\title{
The Hormone Replacement Therapy in Cerebrovascular Diseases: Is the Receptor Remodeled? 四
}

\author{
Yusheng Liu, ,, 1 Shiqin Xu, ${ }^{*}, 1$ Xiaofeng Shen, ${ }^{*}$ Xueneng Guan, ${ }^{\dagger}$ Fuzhou Wang*,
}

EVENTS IN CEREBRAL vessel have long been considered as a leading cause of disability in postmenopausal women with the physiological changes in expression and secretion of sex hormones. Hormone replacement therapy (HRT) emerged as a supplementary therapeutic strategy for them with the potential risk of cerebrovascular accidents. Epidemiological and genetic data showed that an interrelationship exists between hormone replacement and cerebrovascular disorders. Many animal experiments and clinical observations produced different results: these varied from positive to negative. Furthermore, recent studies could not identify the particular hormone, estrogen or progesterone that is more beneficial than the other. Here we summarize the two hormones' effects on cerebrovascular diseases; associated epidemiological and genetic evidences; and the real status of the benefits and risks of HRT as well. We further hypothesize that whatever effects of HRT on brain vessel, hormone receptor expression, density, sensitivity and function may undergo alteration to varying extents, i.e. receptor plasticity give rise to the receptor remodeling in postmenopausal older women, this may terminally produce the unwelcome effects.

\footnotetext{
*: Department of Anesthesiology and Critical Care Medicine, Affiliated Nanjing Maternal and Child Health Care Hospital, Nanjing Medical University, Nanjing, China

†: Neurology Department, Jiangsu Province Hospital of Integrated Traditional and Western Medicine, Nanjing, China

$\Delta$ : Correspondence to Fuzhou Wang, No. 123, Tianfei Xiang, Mochou Rd., Nanjing 210004, China

Tel: +86-25-5222 6112

Fax: +86-25-8420 0723

Email: zfwang50@njmu.edu.cn

1. These authors contributed equally to this work

PQ:Publisher Copyright

Received: 02 April 2013

Revised: 04 June 2013

Accepted: 10 June 2013
}

Keywords: Cerebrovascular disease - Hormone replacement therapy - Estrogen - Progesterone - Inflammation Lipopolysaccharide

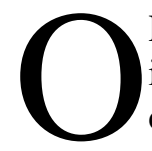
RGANISMS have astounding abilities to recover from considerable external interference and internal imbalance. This potential has been manipulated throughout human history. This ability diminishes with increasing age. As humans age, cerebrovascular diseases have increasingly become one of the leading causes of morbidity and mortality after severe infectious diseases have been controlled. Cerebrovascular accidents, a part of the complex of vascular diseases, have its own origin. While various associated risk factors for stroke have been identified over the past several decades, effective approaches towards preventing this pathological process still remain unclear.

We retrieved PubMed and Google scholar data sources through the keywords of "hormone replacement therapy”, "cerebrovascular disease”, “estrogen" and "progesterone", and combined them together for the second retrieve. All collected data showed that estrogen and progester- one, two members of steroid hormone family, are well known for their roles in cerebrovascular function. But the administration of the two hormones in vascular diseases has been restricted for the severe negative effects attendant to their use. Although the effects of these two molecules have been evaluated and their roles in vascular diseases reviewed, we hypothesize that alteration in hormone receptors plasticity results in the brainvessel-accident during HRT in treated menopausal women. 


\section{Epidemiology for Cerebrovas- cular Diseases}

The World Health Organization (WHO) defines stroke as rapidly developing clinical signs of focal or global disturbance of cerebral function with symptoms lasting 24 hours or longer or leading to death, with no apparent cause except of vascular origin (1). The International Classification of Diseases (ICD) using etiology and pathology has divided stroke into nine major groups. In 1990 the National Institute of Neurological Disorders and Stroke (NINDS) group classified stroke broadly according to its epidemiological, pathological and clinical features (2).

With the development

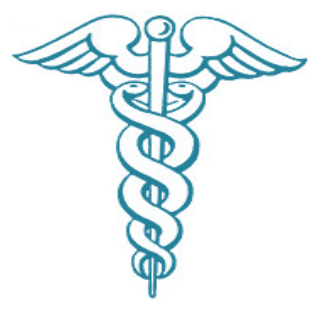

rence of stroke (4). With the use of preventive therapies and interventions, the burden of cerebrovascular diseases is improving dramatically. Educations of the populations at risk play a pivotal role in reducing stroke occurrence. However, the precise mechanisms for cerebrovascular accidents are yet to be clarified.

\section{Genetic Associated Causes of Cerebrovascular Diseases}

Evidence is accumulating indicating that cerebrovascular diseases are strongly associated with genetics. Yamamoto and colleagues (5) reviewed the major causes of cerebrovascular diseases that related directly or indirectly to genetics. Cerebral autosoand utilization of medical imaging equip-ments such as the computerized tomography (CT), magnetic resonance imaging (MRI), position emission tomography (PET) and transcranial Doppler cerebral flow assessment, stroke diagnosis has changed dramatically. With these techniques a far more detailed evaluation can be given, and the prognosis after the attack of the cerebral vascular accident evaluated and improved.

The high mortality of stroke has been recognized worldwide, being higher in the third world than in industrialized countries. Age is the strongest predictor of mortality in stroke-affected individuals. The average mortality rate is $7.6 \%$ for ischemic stroke and $37.5 \%$ for hemorrhagic stroke at 30 days after the accident (3).

In general, the stroke rate changes with race, family history, gender and age. Simultaneously, other so-called modifiable factors such as smoking, obesity, physical activity, hypertension, hyperlipidemia and other risk factors significantly affect the occur- mal dominant arteriopathy with subcortical infarcts and leukoencephalopathy (CADASIL), a stroke syndrome, results from mutation in the gene at Notch 3 encoding for the large Notch 3 transmembrane receptor (6). Normally, Notch 3 comprises of a $97 \mathrm{kDa}$ intracellular fragment and a 210kDa extracellular fragment. However, a large number of the Notch 3 ectodomain $(210 \mathrm{kDa})$ are accumulated within the cerebral vessels in CADASIL patients and models. This strongly suggests that Notch 3 plays a crucial role in CADASIL cases.

X-linked disorders such as Fabry disease are caused by mutation in the $\alpha$-galactosidase A gene. After 3 or 4 decades, patients with Fabry disease suffer stroke, mainly small-vessel ischemic stroke (7). Stroke in such patients is accompanied with hypertension and cardiac abnormalities.

Homocystinuria, an autosomal disorder, has a significant association with the defective gene that normally encodes the cystathio-nine $\beta$-synthase and the deficiency of methylenetetrahydrofolate re-ductase (MTHFR). Furthermore, mitochondrial encephalopathy, lactic acidosis, and strokelike episodes (MELAS) is caused by mutations in mitochondrial DNA (8).

Additionally, sickle cell disease is caused by a mutation in the $\beta$ polypeptide chain of haemoglobin, in which glutamic acid replaces valine. Many such patients suffer their first stroke between the ages of 2 and 5 years (9).

\section{Recovery from Cerebrovascular Diseases}

Although a good number of investigations have been made on cerebrovascular accidents, the results have not been consistently useful. Even so the therapeutic approach to stroke management has seen little change.

Stroke rehabilitation has been defined from the perspectives of different individual structure, process and outcome (10). The basic deficits assessed in stroke comprise motor, sensory, visual and impairments. Many systems exist for assessing stroke patients' abilities to recover. Two most widely used assessments are the Functional Independence Measure (FIM) (11) and the Barthel Activities of Daily Living (LDL) index (12).

There are many major goals of stroke recovery. Rehabilitation from motor deficits is the most common goal: motor deficits are characterized by muscle weakness, clumsiness and fatigue. Individualized therapeutic techniques need to be adapted to patients at different stages of motor recovery (13).

Unfortunately, no consistently effective therapy has been described. Pain in the shoulder of the affected side is very common after brain vessel accidents. Besides, neuropsychological deficits should also be consid- 
ered. Management usually requires an empirical therapist working patiently with verbal or/and non-verbal material and approaches (14).

\section{Basic Actions of Estrogen}

The physiological estrous cycle plays an essential role in regulating secretion and signaling of estrogen: these are functions of the ovaries. Estrogen has the basic molecular structure of perhydrocyclopentanophenanthrene, and exerts its effects in the same way as glucocorticoid. The estrogen receptor is a member of the steroidhormone-receptor family of proteins. It has two structurally distinct receptors, ER- $\alpha$ and ER- $\beta$. Three different receptor complexes exist either as a homodimer $\alpha / \alpha$ or $\beta / \beta$ or a heterodimer $\alpha / \beta$, with each one processing respective unique properties.

When estrogen is transported through the cell membrane, it binds with high affinity to ER. The binding, with the help of several receptor accessory proteins, on the one hand, promotes dissociation of some molecular components of the receptor; on the other hand, it recruits some other assistant proteins to the receptor. Finally, estrogen is carried and translocated into the nucleus where it binds to associated DNA in the domain to elicit appropriate response. Transcription and translation follow. Estrogen increases the expression of vasodilators, promotes the formation of anti-inflammatory molecules and decreases the pro-inflammatory proteins after the challenge of lipopolysaccharide (LPS) in rodent models. As a result, estrogen produces effects on cardiac and cerebral vessels and in inflammatory responses.

Progesterone Functions in Brain Vessel Disorders
A widely accepted function of progesterone is the neuroprotective role in traumatic brain injury. A battery of evidence showed that progesterone functions as a protector in middle cerebral artery occlusion (MCAO) and ischemic brain damage (15-20). However, Murphy demonstrated that progesterone does not ameliorate histological injury after MCAO in previously ovariectomized adult female rats and chronic administration can exacerbate infarction in subcortical regions of such rats (21).

Comparatively, it is not clear whether the benefits of progesterone administration outweigh its advert effects in cerebrovascular diseases (22). The natural progestins, usually progesterone and testosterone, have limited use due to their negative effects. Many new pro-gesterones including nomegestrol acetate (NOMAc) and trimegestone (TMG) that are derived from the parent molecule of progesterone are synthesized (23). Given the fewer side effects, better physiological activities and selfowned pharmacological properties; it is likely that these new derivatives have potential therapeutic values in cerebrovascular disorders.

\section{Estrogen or Progesterone, Which One is Superior?}

Estrogen and progesterone have long been considered as primary hormones in reproductive and maternal behaviors. Emerging data have shown that the two gonadal hormones function as neuro-protective and neuroregenerative agents in traumatic brain injury and stroke. With the progress of many investigations different, even opposite, results have been reported.

While estrogen exerts effect as a prophylactic in the treatment of ischemic brain injury, progesterone exerts effect as a post-injury treat- ment for the acute, ischemic and traumatic injuries of the brain and spinal cord (24). Estrogen plays roles in preserving the antioxidant effect; reducing amyloid-beta (A- $\beta$ ) production and neurotoxicity; increasing in expression of the antiapoptotic factor bcl-2; and activating mitogen activated protein kinase (MAPK) pathways. Progesterone, on the other hand, has a membrane stabilizing effect and suppressing role in neuronal hyperexcitability (25). ZYC-26 (2-(1adamantyl)-4-methylestrone), a nonfeminizing estrogen, takes part in antioxidant activity, rather than ER binding of non-feminizing estrogens, and mediates their potent neuroprotective activity. Additionally, in view of the now known toxicities of chronic feminizing estrogen use in older women, non-feminizing estrogens may be a useful alternative for estrogen-induced brain protection (26). Estrogen provides protective role in increased vulnerability of males and menopausal females to cerebral ischemia by affecting amino acids (27). Transdermal HRT, with 17 -estradiol patch [36 $\mu \mathrm{g} /$ day] plus cyclic oral medroxyprogesterone acetate [2.5 mg/day, for 12 days/month] for 12 months, reduced carotid artery wall thickness, and this reduction may have been induced by an antiatherosclerotic effect combined with the direct effect of estrogen and decreased levels of estrogen-induced Eselectin (28).

Nonetheless, Gordon et al reported that two weeks' pre-treatment with a high physiological dose of $17 \beta$ estradiol increased infarct volume after permanent MCAO (29). This, in contrast, shows that estrogen does have the capacity to promote detrimental actions in the stroke-injured brain. And to some extent, estrogen replacement therapy exacerbates the 
cerebral ischemia-reperfusion (I/R) injury in diabetic ovariectomized female rats through upregulated inflammation after tMCAO (30). In fact, it is possible that the influence of $\mathrm{I} / \mathrm{R}$ on vessel function was more prominent than that of estrogen therapy (31). Moreover, a clinical trial showed that estradiol replacement therapy in elderly women who suffered cerebrovascular disease was unable to protect against reoccurrence of ischemia or to reduce the mortality compared to a placebo (32).

Progesterone exerts its neuroprotective role not merely by interfering with some late pathophysiological mechanisms leading both to selective neuronal damage in the hippocampal CA1 and CA2 subfields, and to shrinkage of the cerebral cortex (33), but also by suppressing specific aspects of the inflammatory response and nitric oxide synthase-2 expression in both permanent and transient ischemia (34). Moreover, synthetic progestins caused endothelial disruption, accumulation of monocytes in the vessel wall, platelet activation and clot formation. All these actions seem to be associated with combined administration of estrogen and progestin (35). As such, the therapeutic strategies on cerebrovascular diseases should be weighed wisely. Using progesterone with minimal vascular toxicity might result in much safer estrogen preparations for those people at risk of cerebral vessel accidents.

Inflammatory responses have been proved to participate in neuropathological changes. Estrogen functions in these through regulating the expression of adhesion molecules, inducible nitric oxide synthase (iNOS) and other inflammatory proteins (36), and protecting the cerebral blood vessels from endothelial dysfunction induced by A- $\beta$ in Alzheimer's dis- ease (AD) (37). Furthermore, 17 $\beta$ estradiol favorably affects vascular inflammation processes by affecting high sensitivity C-reactive protein (hs-CRP) and reducing cell adhesion molecules (38). Estrogen (17 $\beta$ estradiol) protects infarcted cerebral cortex by decreasing heat shock protein 27 (HSP-27) synergistically with 1 a,25-(OH)2-vitamin-D3 (1,25-D3) (39); inhibits the migration of inflammatory cell, particularly granulocytes, into the rat carotid arteries after acute endoluminal injury; but the progestin medroxypro-gesterone acetate (MPA), a synthetic analog of progesterone, blocks this effect (40), attenuates blood-brain barrier (BBB) disruption by down-regulation of matrix metalloproteinases (MMP2 and MMP9) expression after I/R injury (41). Meanwhile, the presence of progesterone would diminish the antiinflammatory effects of estrogen in ischemic brain injury after the rodent is challenged with LPS (42). The effect of estrogen or progesterone in the inflammatory process occurring after cerebrovascular accidents remain to be proved and may need large-sample, evidence-based and randomized clinical trials and meta-analysis.

In addition to estrogen and progesterone, testosterone may have a beneficial effect on axonal regeneration after injury in the central nervous system. Testosterone replacement post-MCAO accelerated functional recovery in castrate rats, suggesting a potential therapeutic role for testosterone replacement in stroke recovery (43).

Brass postulated that only estrogen replacement therapy in postmenopausal women is not effective in reducing the risk of a first or a recurrent stroke or death with established vascular disease. In contrast, there may be an increased risk of fatal stroke among those who taking HRT (44). Consistent with a meta-analysis on the association between HRT and subsequent stroke, the use of HRT would increase the risk of stroke, typically ischemic cerebrovascular events (45).

While these literatures present a contrary concept against conventional HRT in postmenopausal women, more recent research put a shine on it, i.e. show no significant association between hormone therapy and risk of total stroke in women during 10.5 years follow-up, but the preparations of estrogen and time for initiation of treatment might affect the risk of stroke (46). A lifetime exposure to ovarian estrogens may protect against non-cardioembolic ischemic stroke, but a very early age of exposure initiation could be disadvantageous (47). Combination administration of estradiol valerate/medroxyprogesterone acetate (E(2)V/MPA) over nine years and at follow-up one year after discontinuation showed that lower dosages of HRT were as effective as higher doses in improving climacteric symptoms and health-related quality of life ratings and had fewer safety concerns (48).

It is certain that we could say that HRT has mixed results. Different studies arrived at different conclusions for subjective or objective reasons. Opposite results got from animal experiments or clinical trials notwithstanding, and even though some scientists suggested giving up the HRT $(49,50)$, the benefits of HRT is clear though the associated risks are yet to be fully elucidated. As estrogen and progesterone might function alone or together in cerebral vessel conditions, decisions in sup- 
port or against their use in vascular associated events in the elderly must be considered carefully.

\section{Is the Receptor Remodeled?}

No matter which hormone, the first step for its role in peripheral organs is the binding to its receptor. The levels of estrogen and progesterone in circulation vary in different stages of periand post-menopausal women. The conventional clinical practice mainly concentrates on the administration of these hormones as the so-called HRT. Although the therapy can produce certain positive effects on acute cerebral injury, whether long-term exposure to such hormones is overall beneficial or not is questionable. We hypothesize that the expression of the receptors to these hormones is changed in the post menopausal elderly, especially at the time of and in the presence of stroke or I/R. Along with this, the plasticity of hormone receptor may alter and result in a corresponding shift in its sensitivity to ligands and down-regulation of its function. All these may be the receptor remodeling that result in dysfunction of the receptors. Besides this, the targeting direction of hormones made a counter-clockwise change to produce target-directed functional alteration of HRT hormones. As a result of this, the HRT showed an unacceptable effect. In sum, the disappointing result of HRT may be from the remodeling of hormone receptor in targeted position, especially in the cerebrovascular system in menopausal women.

Testing a hypothesis is always complex and challenging thing because of the difficulty in replicating the clinical environment, design corresponding study-protocols as well as the influence of subjective bias of researchers. For this receptor remod- eling theory, we could perform testing via a combination of ways including both clinical trials and basic experiments.

On the one hand, clinical trials are mainly for the test of the sensitivity of receptors to their ligands. This may be associated with the neuroendocrinological system, and hormone circadian balance. In further, multicentered, global wide and double blinded trials are necessary to avoid $\beta$ error (i.e. increase the power of the study $1-\beta$ ) in the study for various subjective and objective reasons.

On the other hand, basic experiments would provide adequate evidence for this hypothesis. Firstly, identifying test models similar to man is the first problem to solve. We can use the higher mammals such as monkey and gorilla as our experimental objects; of course this should be approved by animal protection committee for research.

Secondly, the genetic alteration of receptor at and after menopause could be studied with gene microarray and plasmid transfection.

Thirdly, the binding ability of hormones to their receptors and sensitivity could be tested by in vitro cell culture and transgenetic or gene knockout cell stains. In addition to this, the co-focal microscopy and radioimmunoassay are suitable for analyzing the translocation of HRT hormones into nuclei and binding to special sites and the targeted change of receptor and hormones. To approve this study,

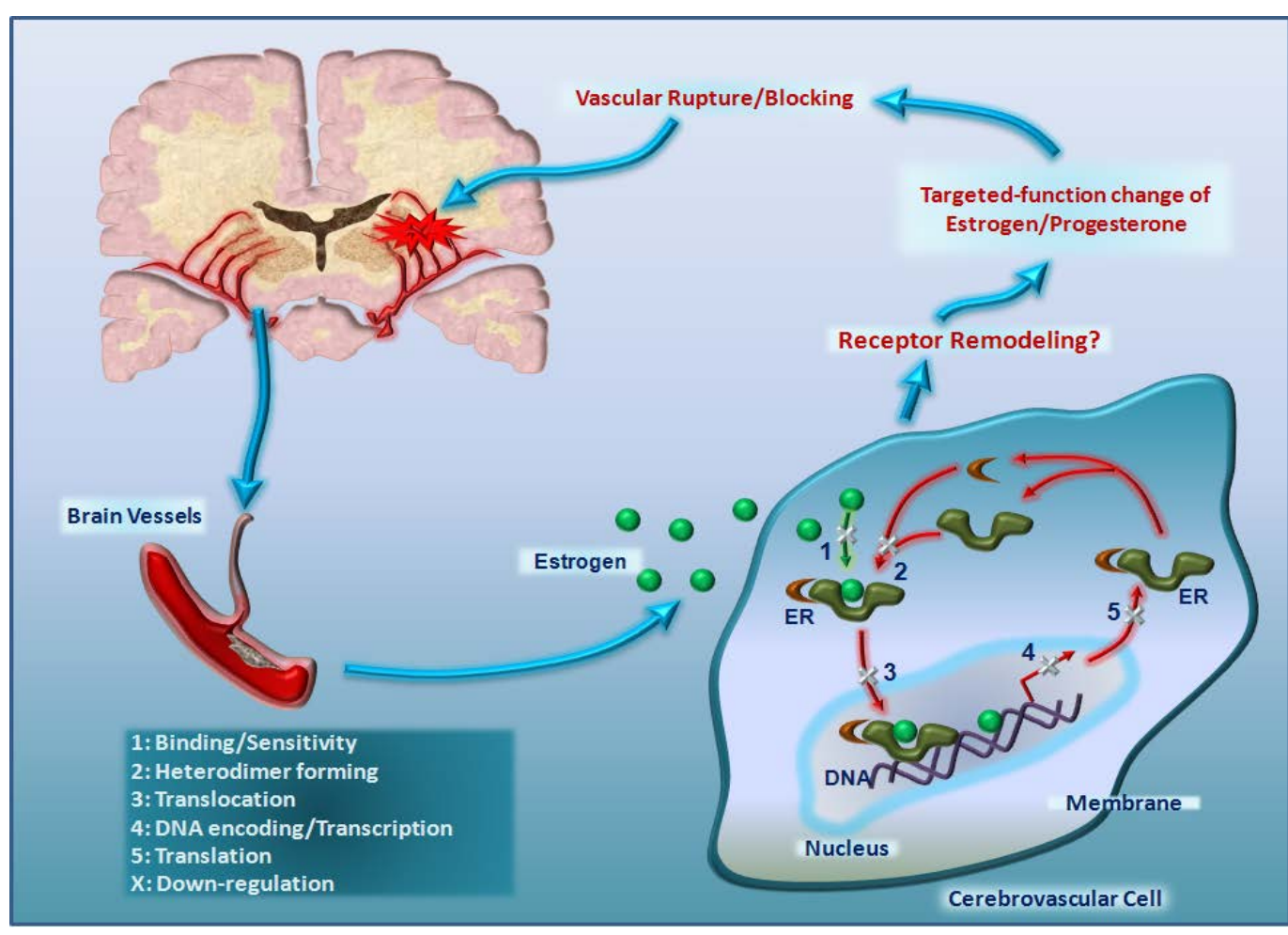

Figure 1: Schematic diagram of the hormone receptor remodeling in menopausal brain. If the fragile brain vessels in postmenopausal women confront a large body of gonadal hormones, the down-regulated hormone receptors resulting from the ovaries' inability to produce eggs cannot satisfy the binding requirement of hormones; and their changed sensitivity to the ligands (numbered 1); which leads to the difficulty in formation of receptor-dimer complex (numbered 2); and followed by translocation failure in hormone-receptor complex into the nucleus (numbered 3); down-stream this produce a halt in processes such as gene encoding/transcription (numbered 4) and translation (numbered 5). The aforementioned series events in cerebrovascular cells change the plasticity of sex hormones receptors, which was dubbed as "the hormone-receptor remodeling" in menopausal population, and this ultimately produce a targeted-functional alteration of hormones, which either exert function in other peripheral end-organs or lead to brain vascular accidents. 
a detailed study protocol should be produced and a step-by-step procedure used. It is only by doing so that a reasonable and reliable result on whether the present hypothesis is right or not could be determined.

Any paradoxical results from different studies need to be explained, no matter what theories or models are used. For the present hypothesis on HRT in cerebrovascular diseases, if it is proved, at least in part, the benefits are significant. In addition to giving explanation to this ironic phenomenon, the more direct effects are that we can take certain steps to find useful therapeutic methods to treat it. It would be a new start point to find ways to resolve such problems as: 1) How to improve the sensitivity of HRT hormone receptors to their ligands in postmenopausal populations? 2) How to avoid the genetic alteration in these receptor-associated genes encoding? 3) Are there any blockade factors existing that interrupt the normal process of hormone's action? 4) Will the existence of this HRT remodeling theory be beneficial or not?

The administration of HRT in postmenopausal people, if the present hypothesis is proven true, would be reevaluated; its positive and negative effects better understood. If it is determined that it was wrong originally to treat these people with HRT, a more reliable treatment may be put proposed and explored.

\section{Conclusion}

There is a need for a better understanding of the role of hormones in cerebrovascular events. It is ironic that on one hand HRT provides neuroprotective effects on menopausal women after or during a stroke, but on the other hand is also something of a problem in this context.
Past knowledge should help to intelligently guide the future. No matter the results one obtains from HRT, further exploration should be processed to clarify the exact role of hormones in cerebrovascular-risk threatening the elderly. Possible answers to this question may come from extending the research, where new roles for hormones in cerebrovascular diseases are prime objectives. The remodeling of hormone receptor, a consequence of the alteration in receptor plasticity resulting from the expression, density, sensitivity and function, may give rise to a target-directed change in HRT hormones. Ultimately, we could not see what we want to see. If the present hypothesis is true, it would explain why so many different results were obtained under similar conditions, and additionally give impetus for further research into this problem with a view to finding corresponding interventional methods.

\section{List of Abbreviations}

$A D$, Alzheimer's disease; A- $\beta$, Amyloidbeta; BBB, Blood-brain barrier; HSP-27, Heat shock protein 27; hs-CRP, High sensitivity C-reactive protein; HRT, Hormone replacement therapy; iNOS, Inducible nitric oxide synthase; $1 / R$, Ischemia/reperfusion; LPS, Lipopolysaccharide; MMP, Matrix metalloproteinases; MPA, Medroxyprogesterone acetate; MCAO Middle cerebral artery occlusion; MAPK Mitogen activated protein kinase.

\section{Acknowledgements}

This work is supported by the National Natural Scientific Foundation of China (NSFC, 30901397, 81271242); Nanjing Medical University Grant for Science \& Technology Development (08NMUZ-033); Nanjing Municipal Young Scientist Grant (201208009); and Nanjing Municipal Developmental Key Grant of Medical Science (ZKX10018).

\section{Conflict of Interests}

\section{None}

\section{References}

1. Burke D, Wissel J, Donnan GA Pathophysiology of spasticity in stroke. Neurology 2013; 80: S20-6.
2. Chen $P H$, Gao S, Wang $Y J, X u A D$, Li YS, Wang D. Classifying Ischemic Stroke, from TOAST to CISS. CNS Neurosci Ther 2012;18: 452-6.

3. Norrving B, Kissela $B$. The global burden of stroke and need for a continuum of care. Neurology 2013;80: S5-12.

4. Della-Morte D, Guadagni F, Palmirotta R, Testa G, Caso V, Paciaroni $\mathrm{M}$, Abete $\mathrm{P}$, Rengo $\mathrm{F}$, Ferroni P, Sacco RL, Rundek T. Genetics of ischemic stroke, strokerelated risk factors, stroke precursors and treatments. Pharmacogenomics 2012; 13: 595-613.

5. Yamamoto $Y$, Craggs L, Baumann M, Kalimo H, Kalaria RN. Review: molecular genetics and pathology of hereditary small vessel diseases of the brain. Neuropathol Appl Neurobiol 2011; 37: 94-113.

6. Arboleda-Velasquez JF, Manent J, Lee JH, Tikka S, Ospina C Vanderburg CR, Frosch MP, Rodríguez-Falcón M, Villen J, Gygi S, Lopera F, Kalimo H, Moskowitz MA, Ayata C, Louvi A, ArtavanisTsakonas S. Hypomorphic Notch 3 alleles link Notch signaling to ischemic cerebral small-vessel disease. Proc Natl Acad Sci USA 2011; 108: E12835.

7. Romão $E A$, Lourenço $C M$, Júnior WM, Rolfs A, Muñoz V, Vieira Neto OM, Dantas M, Silva GE, Costa RS. What lies beneath: Fabry nephropathy in a female patient with severe cerebrovascular disease. Clin Nephrol 2013; 79: 330-4.

8. Parlakis SG, Phillips PC, Dimauro S, De Vivo DC, Rowland LP. Mitochondrial myopathy, encephalopathy, lactic acidosis, and stroke-like episodes: a distinctive clinical syndrome. Ann Neurol 1984; 16: 481-8.

9. Flanagan JM, Sheehan V, Linder $\mathrm{H}$, Howard TA, Wang YD, Hoppe CC, Aygun B, Adams RJ, Neale GA, Ware RE. Genetic mapping and exome sequencing identify 2 mutations associated with stroke protection in pediatric patients with sickle cell anemia. Blood 2013; 121: 323745.

10. DiCocco JM, Fabian TC, Emmett KP, Magnotti LJ, Zarzaur BL, Khan N, Kelly JM, Croce MA. Functional outcomes following blunt cerebrovascular injury. J Trauma Acute Care Surg 2013; 74: 955-60.

11. Unsworth CA. Selection for rehabilitation: acute care discharge patterns for stroke and orthopaedic patients. Int J Rehabil Res 2001; 24: 103-14.

12. Collin C, Wade DT, Davis S, Horne V. The Barthel ADL Inndex: a reliability study. Int Disabil Stud 1988; 10: 61-3.

13. Dimyan MA, Cohen LG. Neuroplastic- 
ity in the context of motor rehabilitation after stroke. Nat Rev Neurol 2011; 7: 76-85.

14. Gross BA, Du R. Cerebrovascular neurosurgery 2011. J Clin Neurosci 2012; 19: 1344-7.

15. Debert CT, Ide K, Poulin MJ. Effects of estrogen and progesterone on cerebrovascular responses to euoxic hypercapnia in women. Climacteric 2012; 15: 621-31.

16. Sayeed I, Guo Q, Hoffman SW, Stein DG. Allopregnanolone, a progesterone metabolite, is more effective than progesterone in reducing cortical infarct volume after transient middle cerebral artery occlusion. Ann Emerg Med 2006; 47: 381-9.

17. Cerrantes M, Gonzalez-Vidal MD, Ruelas R, Escobar A, Moralí G. Neuroprotective effects of progesterone on damage elicited by acute global cerebral ischemia in neurons of the caudate nucleus. Arch Med Res 2002; 33: 6-14.

18. Krause DN, Duckles SP, Pelligrino DA. Influence of sex steroid hormones on cerebrovascular function. J Appl Physiol 2006; 101: 1252-61.

19. Gibson CL, Murphy SP. Progesterone enhances functional recovery after middle cerebral artery occlusion in male mice. J Cereb Blood Flow Metab 2004; 24: 805-13.

20. Murphy SJ, Littleton-Kearney MT, Hurn PD. Progesterone administration during reperfusion, but not preischemia alone, reduces injury in ovariectomized rats. J Cereb Blood Flow Metab 2002; 22: 1181-8.

21. Murphy SJ, Traystman RJ, Hurn PD. Progesterone exacerbates striatal stroke injury in progesterone-deficient female animals. Stroke 2000; 31: 1173-8.

22. Burkman RT. Hormone replacement therapy. Current controversies. Minerva Ginecol 2003; 55: 107-16.

23. Sitruk-Ware R. New progestagens for contraceptive use. Hum Reprod Update 2006; 12: 169-78.

24. Herson PS, Koerner IP, Hurn PD. Sex, sex steroids, and brain injury. Semin Reprod Med 2009; 27: 229-39.

25. Roof RL, Hall ED. Gender differences in acute CNS trauma and stroke: neuroprotective effects of estrogen and progesterone. J Neurotrauma 2000; 17: 367-88.

26. Patkar S, Farr TD, Cooper E, Dowell FJ, Carswell HV. Differential vasoactive effects of oestrogen, oestrogen receptor agonists and selective oestrogen receptor modulators in rat middle cerebral artery. Neurosci Res 2011; 71: 78-84.

27. Cimarosti $H$, Siqueira IR, Zamin LL, Nassif M, Balk R, Frozza R, Dalmaz C, Netto CA, Salbego C.
Neuroprotection and protein damage prevention by estradiol replacement in rat hippocampal slices exposed to oxygen-glucose deprivation. Neurochem Res 2005; 30: 583-9.

28. Sumino H, Ichikawa $S$, Kasama $S$, Kumakura H, Takayama Y, Sakamaki T, Kurabayashi M. . Effect of transdermal hormone replacement therapy on carotid artery wall thickness and levels of vascular inflammatory markers in postmenopausal women. Hypertens Res 2005; 28: 579-84.

29. Gordon KB, Macrae IM, Carswell HVO. Effects of $17 \beta$-oestradiol on cerebral ischaemic damage and lipid peroxidation. Brain Res 2005; 1036: 155-62.

30. Yong Y, Xie HJ, Zhang YF, Yang QD Liao DF, Yang HL, Yan PK, Liu ZJ. $17 \beta$-estradiol potentiates ischemiareperfusion injury in diabetic ovariectomized female rats. Brain Res 2005; 1054: 192-9.

31. Suzuki S, Brown CM, Wise PM. Neuroprotective effects of estrogens following ischemic stroke. Front Neuroendocrinol 2009; 30: 201-11.

32. Stein DG. Brain damage, sex hormones and recovery: a new role for progesterone and estrogen? Trends Neurosci 2001; 24: 386-91.

33. Moralí G, Letechipía-Vallejo G, López-Loeza $E$, Montes $P$, Hernández-Morales L, Cervantes M. Postischemic administration of progesterone in rats exerts neuroprotective effects on the hippocampus. Neurosci Lett 2005; 382: 286-90.

34. Gibson CL, Constantin D, Prior MJW, Bath PM, Murphy SP. Progesterone suppresses the inflammatory response and nitric oxide synthase-2 expression following cerebral ischemia. Exp Neurol 2005; 193: 522-30.

35. Thomas T, Rhodin J, Clark L, Garces A. Progestins initiate adverse events of menopausal estrogen therapy. Climacteric 2003; 6: 293-301.

36. Pelligrino DA, Galea E. Estrogen and cerebrovascular physiology and pathophysiology. Jpn J Pharmacol 2001; 86: 137-58.

37. Singh M, Simpkins JW, Simpkins JW, Bimonte-Nelson HA, Bimonte-Nelson HA, Brinton RD, Brinton RD. Window of opportunity for estrogen and progestin intervention in brain aging and Alzheimer's disease. Brain Res 2013 1514: 1-2.

38. Stork S, von Schacky C, Angerer P. The effect of 17 beta-estradiol on endothelial and inflammatory markers in postmenopausal women: a randomized controlled trial. Atherosclerosis 2002; 165: 301-7.

39. Losem-Heinrichs E, Görg B, Redecker C, Schleicher A, Witte OW, Zilles K, Bidmon HJ. 1a,25-
Dihydroxy-vitamin D3 in combination with $17 \beta$-estradiol lowers the cortical expression of heat shock protein-27 following experimentally induced focal cortical ischemia in rats. Arch Biochem Biophys 2005; 439: 70-9.

40. Xing D, Miller A, Novak L, Schleicher A, Witte OW, Zilles K, Bidmon HJ. Estradiol and progestins differentially modulate leukocyte infiltration after vascular injury. Circulation 2004; 109: 234-41.

41. Liu R, Wen Y, Perez E, Wang X, Day AL, Simpkins JW, Yang SH. 17ßEstradiol attenuates blood-brain barrier disruption induced by cerebral ischemia-reperfusion injury in female rats. Brain Res 2005; 1060: 55-61.

42. Sunday L, Tran MM, Krause DN, Duckles SP. Estrogen and progesterones differentially modulate vascular proinflammatory factors. Am J Physiol Endocrinol Metab 2006; 291: E261-7.

43. Pan $\mathrm{Y}$, Acharya $A B$, Patrick $P H$, Patrick $\mathrm{PH}$, Oliver $\mathrm{D}$, Morley JE. Effect of testosterone on functional recovery in a castrate male rat stroke model. Brain Res 2005; 1043: 195-204.

44. Brass LM. Hormone replacement therapy and stroke clinical trials review. Stroke 2004; Suppl I: 2644-7.

45. Bath PMW, Gray LJ. Association between hormone replacement therapy and subsequent stroke: a metaanalysis. Br Med J 2005; 330: 342-5.

46. Li C, Engstrom G, Hedblad B, Berglund $G$, Janzon L. Risk of stroke and hormone replacement therapy. A prospective cohort study. Maturitas 2006; 54: 11-8.

47. de Lecinana MA, Egido JA, Fernandez C, Martínez-Vila E, Santos S, Morales A, Martínez E, Pareja A, Alvarez-Sabín J, Casado I; PIVE Study Investigators of the Stroke Project of the Spanish Cerebrovascular Diseases Study Group. Risk of ischemic stroke and lifetime estrogen exposure. Neurology 2007; 68: 33-8.

48. Heikkinen J, Vaheri R, Timonen U. A 10-year follow-up of postmenopausal women on long-term continuous combined hormone replacement therapy: Update of safety and qualityof-life findings. $\mathrm{J} \mathrm{Br}$ Menopause Soc 2006; 12: 115-25.

49. Couzin J. The great estrogen conundrum. Science 2003; 302: 1136-8.

50. Wickelgren I. Brain researchers try to salvage estrogen treatment. Science 2003; 302: 1138-9.

\section{SCIENCE INSIGHTS ${ }^{\circledR}$}

WWW.BONOI.ORG

ISSN: 2329-5856 (online) 